\title{
¿Está exenta de consecuencias adversas la perforación iatrogénica de la vesícula biliar durante la colecistectomía laparoscópica electiva?
}

\author{
Elías Domínguez-Comesaña ${ }^{1}$, Elías Domínguez-Fernández y Rubén Domínguez-Fernández ${ }^{2}$
}

Iatrogenic gallbladder perforation during elective laparoscopic cholecystectomy, is it exempt from adverse consequences?

\begin{abstract}
Aim: This study was conducted to evaluate the outcomes in patients with iatrogenic gallbladder perforation (IGP), investigating its association with postoperative complications, use of antibiotics and drains, operative time and postoperative stay. Materials and Method: 1703 patients who underwent elective laparoscopic cholecystectomy were included and divided in two groups: with IGP (Group 1, $\mathrm{n}=321$ ) and without IGP (Group 2, $\mathrm{n}=1382$ ). We compared the outcomes between both groups. Results: The IGP rate was $18.85 \%$. Isolated bile spillage occurred in 241 patients $(14.15 \%)$, and stone spillage in 80 patients $(4.64 \%)$. The incidence of surgical site infections was not different between both groups. The IGP rate was significantly higher in male $(43.3 \%$ vs $31.3 \%)$, in patients with perivesicular adhesions $(17.75 \%$ vs $10.5 \%)$ and in patients with histologic diagnosis of acute cholecystitis (11.52\% vs $4.92 \%)$. Operative time was significantly longer in patients with IGP (77.3 vs 65.4 minutes). Intraoperative drain and antibiotic use, as well as postoperative stay were, also, significantly higher in patients with IGP. There was not any late complication. Conclusion: Bile and gallstones spillage do not lead to an increase in surgical site infections, but is associated with an increased use of antibiotics and drains, longer operative time and longer postoperative stay.

Key words: iatrogenic gallbladder perforation; surgical site infection; laparoscopic cholecystectomy.
\end{abstract}

\section{Resumen}

Objetivo: Este estudio se llevó a cabo para valorar las consecuencias de la perforación iatrogénica de la vesícula biliar (PIVB), investigando su asociación con complicaciones posoperatorias, uso de antibióticos y drenajes, duración de la cirugía y estancia posoperatoria. Materiales y Método: Se incluyeron 1.703 pacientes con colecistectomía laparoscópica electiva, divididos en dos grupos: con PIVB (Grupo 1; $\mathrm{n}=321$ ) y sin PIVB (Grupo 2; $\mathrm{n}=1.382$ ). Se compararon los resultados entre ambos grupos. Resultados: El porcentaje de PIVB fue de 18,85\%. El vertido aislado de bilis ocurrió en 241 pacientes $(14,15 \%)$ y el de bilis y cálculos en 80 pacientes $(4,64 \%)$. La incidencia de infección del sitio quirúrgico no fue diferente entre ambos grupos. La incidencia de PIVB fue mayor en varones $(43,3 \%$ vs $31,3 \%$ ), pacientes con adherencias perivesiculares (17,75\% vs. 10,5\%) y pacientes con diagnóstico histológico de colecistitis aguda (11,52\% vs. 4,92\%). La PIVB se asoció significativamente con mayor duración de la cirugía (77,3 vs. 65,4 minutos), mayor uso de drenajes y antibióticos y mayor estancia posoperatoria. No hubo complicaciones tardías. Discusión y Conclusión: La PIVB no aumenta la incidencia de infección, pero se asocia con un mayor uso de drenajes y antibióticos, mayor duración de la cirugía y mayor estancia posoperatoria.

Palabras clave: perforación iatrogénica de la vesícula biliar; infección del sitio quirúrgico; colecistectomía laparoscópica. 


\section{Introducción}

La colecistectomía laparoscópica (CL) es desde hace casi tres décadas, el método de elección para el tratamiento quirúrgico de la colelitiasis sintomática. En este procedimiento, la disección de la vesícula se lleva a cabo, generalmente, mediante el uso de coagulación monopolar y es necesario sujetar y mover la vesícula con la ayuda de pinzas. Por ello, debido a que la pared vesicular es fina, no es infrecuente la perforación iatrogénica de la misma, con una incidencia que oscila entre el $10 \%$ y el $40 \%$ de los pacientes ${ }^{1-3}$. Como resultado de esta complicación se produce el vertido de bilis $\mathrm{y}$, en ocasiones, la salida de cálculos hacia el peritoneo. Aunque la mayoría de los cálculos que permanecen retenidos en la cavidad peritoneal no causan problemas, se han descrito muchas complicaciones, tales como abscesos intra o retroperitoneales y formación de tractos fistulosos ${ }^{1,3-5}$. Sin embargo, el vertido aislado de bilis no conduce a un aumento de las infecciones del sitio quirúrgico (ISQ) $)^{1,6-8}$, a pesar de que se ha descrito que $22 \%$ a $37,9 \%$ de los pacientes tienen bacterobilia ${ }^{9,10}$. Hay muy pocas referencias en la literatura médica sobre la relación entre la PIVB y el tiempo operatorio y la estancia posoperatoria ${ }^{11} \mathrm{y}$, hasta donde alcanza nuestro conocimiento, no hay publicaciones previas en las que se analice la relación de la PIVB con la utilización de antibióticos y drenajes quirúrgicos. El objetivo de este estudio es valorar la relación entre la PIVB y el uso de antibióticos y drenajes, así como su influencia en la duración de la cirugía y la incidencia de complicaciones posoperatorias.

\section{Materiales y Método}

En el estudio se incluyeron 1.703 pacientes que experimentaron una $\mathrm{CL}$ electiva por colelitiasis sintomática entre enero de 2005 y mayo de 2018. Los pacientes fueron divididos en dos grupos: con PIVB (grupo 1) y sin PIVB (grupo 2). Se excluyeron del estudio los pacientes menores de 18 años y los que habían sido operados de forma urgente. Los datos fueron obtenidos a partir de una base de datos prospectiva iniciada en enero de 2005. La CL se llevó a cabo utilizando cuatro trocares; la vesícula se diseccionó utilizando coagulación monopolar, bien con gancho o bien con tijeras y se extrajo de la cavidad peritoneal a través de la incisión periumbilical, en el interior de una bolsa de plástico. En los pacientes en los que se produjo PIVB, se lavó localmente el peritoneo con suero salino, se aspiró la bilis y se intentó recuperar los cálculos vertidos. Se hizo profilaxis antimicrobiana cuando estaba indicado, utilizando, en la mayoría de los casos, una combinación de amoxicilina ( $2 \mathrm{~g}$ ) y ácido clavulánico $(200 \mathrm{mg})$, administrados por vía endovenosa en los 30-60 minutos que precedían a la cirugía. En los pacientes en los que no se había prescrito profilaxis antimicrobiana preoperatoria, se administró un antibiótico intraoperatorio, a criterio del cirujano, en caso de hallazgos sugestivos de colecistitis o en caso de PIVB. Tras la cirugía, los pacientes fueron examinados diariamente hasta el alta hospitalaria, y a los 30 días de la misma, de forma ambulatoria. Además, en junio de 2018 se examinaron los historiales clínicos de los pacientes en los que se había producido vertido de cálculos hacia la cavidad peritoneal. La ISQ se diagnosticó siguiendo las recomendaciones de los Centros para Control y Prevención de Enfermedades ${ }^{12}$. El análisis estadístico se llevó a cabo utilizando el paquete estadístico SPSS v.20.0 para Mac (Statistical Package for Social Sciences Inc., Chicago, Illinois, USA). Los valores se expresaron como medias con desviación estándar, medianas con rango intercuartílico o recuentos y porcentajes, según fuera apropiado. Para evaluar las diferencias entre los dos grupos principales se utilizaron el test de Mann-Withney, para comparar variables cuantitativas, y los tests de Fisher o $\chi 2$, para comparar variables cualitativas. Se aceptó la significación estadística para valores de $\mathrm{p}$ $<0,05$. El estudio fue llevado a cabo de acuerdo con los principios éticos recogidos por la Declaración de Helsinki y fue aprobado por el comité local de investigación.

\section{Resultados}

Durante el periodo de tiempo estudiado, 1.916 pacientes experimentaron una CL por colelitiasis sintomática. De ellos, 213 fueron excluidos del estudio, 202 debido a que las variables intraoperatorias analizadas no fueron registradas adecuadamente por el cirujano y 11 debido a que tenían menos de 18 años de edad. De los 1.703 pacientes incluidos en el estudio, $572(33,6 \%)$ eran hombres y 1.131 $(66,4 \%)$ mujeres. La edad media de los pacientes fue de 56,1 \pm 16 años. El grupo 1 (con PIVB) incluía 321 pacientes $(18,8 \%$ ) y el grupo 2 (sin PIVB) 1.382 $(81,2 \%)$. El vertido aislado de bilis ocurrió en 241 $(14,2 \%)$ pacientes, mientras que el vertido de bilis y cálculos ocurrió en $80(4,6 \%)$; en $14(0,8 \%)$ pacientes no se consiguió extraer todos los cálculos extravasados al peritoneo. Se administró profilaxis anti- 
Tabla 1. Comparación entre los pacientes con perforación iatrogénica de la vesícula biliar (grupo 1) y los pacientes sin perforación iatrogénica de la vesícula biliar (grupo 2)

\begin{tabular}{|lccc|}
\hline Variables & Grupo 1 (n) & Grupo 2 (n) & P \\
\hline Varón/mujer (n) & $139 / 182$ & $433 / 949$ & 0,001 \\
\hline Edad media \pm DE & $57,4 \pm 16$ & $55,8 \pm 16$ & 0,313 \\
IMC medio \pm DE & $29,3 \pm 5$ & $28,9 \pm 5$ & 0,577 \\
ASA I-II/ASA III-IV (n) & $262 / 59$ & $1.181 / 201$ & 0,432 \\
Adherencias perivesiculares, sí/no & $57 / 264$ & $145 / 1.237$ & 0,002 \\
Diagnóstico histológico de colecistitis aguda, sí/no & $37 / 284$ & $68 / 1.314$ & 0,001 \\
Antibiótico intraoperatorio, sí/no & $60 / 43$ & $58 / 357$ & 0,001 \\
Tasa de conversión & $12 / 309$ & $64 / 1.318$ & 0,212 \\
Tiempo operatorio \pm SD (min) & $77,3 \pm 33,6$ & $65,4 \pm 34,6$ & 0,001 \\
Residente, sí/no & $38 / 283$ & $145 / 1.236$ & 0,162 \\
Drenaje, sí/no & $111 / 210$ & $291 / 1.091$ & 0,001 \\
Complicaciones posoperatorias, sí/no & $28 / 293$ & $154 / 1.228$ & 0,116 \\
Infección del sitio quirúrgico, sí/no & $11 / 310$ & $79 / 1.303$ & 0,114 \\
Estancia posoperatoria \pm DE (días) & $2,6 \pm 3,6$ & $2,4 \pm 3,6$ & 0,002 \\
\hline
\end{tabular}

microbiana preoperatoria en 218 pacientes del grupo $1(68 \%)$ y en 967 pacientes del grupo $2(70 \%)$. Se administró una dosis intraoperatoria de antibiótico en 118 pacientes que no habían recibido profilaxis preoperatoria, 60 del grupo $1(58,3 \%)$ y 58 en el grupo $2(14 \%)(p=0,001)$. Cuarenta y tres pacientes del grupo $1(13,4 \%)$ y 467 del grupo $2(33,4 \%)$ no recibieron ningún antibiótico. La incidencia de complicaciones posoperatorias no fue significativamente diferente entre los pacientes con o sin PIVB $(8,7 \%$ y $11,4 \%$, respectivamente; $p=0,116$ ). La incidencia de ISQ tampoco fue significativamente diferente entre ambos grupos ( $3,4 \%$ y $5,7 \%$, respectivamente; $\mathrm{p}=0,114)$. Entre los pacientes que no recibieron ningún antibiótico tampoco hubo diferencias significativas en la incidencia de ISQ $(2,9 \%$ y $4,3 \%$, respectivamente; $p=0,230$ ). Tampoco hubo diferencias significativas en la incidencia de ISQ si se compararon los pacientes sin PIVB y los pacientes con extravasación de cálculos $(5,7 \%$ y $3,5 \%$, respectivamente; $p=0,231)$. En el grupo de 14 pacientes en los que no se consiguió extraer todos los cálculos extravasados no hubo ninguna complicación tardía, con una media de seguimiento de 50,6 meses (rango de 5 a 136) (Tabla 1).

La incidencia de PIVB fue significativamente mayor en varones, pacientes con adherencias perivesiculares y pacientes con diagnóstico histológico de colecistitis aguda. Como se puede apreciar en la
Tabla 1, la duración de la cirugía, el uso de drenajes y el uso de antibióticos fueron significativamente mayores en los pacientes con PIVB.

\section{Discusión}

En la literatura médica se describen incidencias de PIVB que varían entre el $10 \%$ y el $40 \%^{1-3}$, mientras que la incidencia de extravasación de cálculos se sitúa entre el $2 \%$ y el $20 \%^{11,13}$. En nuestro estudio, la incidencia de PIVB fue de $18,9 \%$ y la de extravasación de cálculos, de 4,6\%. Cuando se produce la apertura accidental de la vesícula biliar, con extravasación de bilis y/o cálculos, la mayor parte de los cirujanos recurren a la irrigación y aspiración con suero salino, con el fin de limpiar el peritoneo y facilitar la búsqueda de los cálculos. Además, algunos cirujanos administran antibióticos intraoperatorios si el paciente no había recibido previamente profilaxis antimicrobiana. En nuestro estudio, el uso de antibióticos intraoperatorios fue significativamente mayor en pacientes con PIVB (58\% vs. 14\%). Debido al hecho de que, en ausencia de infección aguda o de instrumentación de la vía biliar, sólo $4 \%$ de los pacientes tienen cultivos de bilis positivos ${ }^{14}$, la mayoría de los autores creen que no es necesario administrar antibióticos en los pacientes con PIVB ${ }^{15,16}$. De hecho, en nuestro es- 
tudio la incidencia de ISQ, tanto superficial como profunda, no fue diferente entre los pacientes con o sin PIVB, incluso entre los que no recibieron ningún antibiótico, y otros autores han obtenido conclusiones similares $^{6-8,11}$. Cuando se produce una perforación accidental de la vesícula, es frecuente la extravasación de cálculos; en nuestra serie ocurrió en $25 \%$ de los pacientes, que es una incidencia inferior a la comunicada por otros autores ${ }^{17}$. En nuestro estudio no hubo diferencias entre los pacientes con o sin extravasación de cálculos con respecto a la incidencia de ISQ y no hemos encontrado referencias en los artículos publicados previamente. Un problema diferente es el de los cálculos no recuperados, ya que hay bastantes publicaciones en las que se describe una incidencia de complicaciones del $0,04 \%$ al $19 \%^{5}$; se han descrito casos de abscesos intraabdominales y retroperitoneales, así como fistulización a la pared abdominal. La incidencia de cálculos no recuperados oscila entre el 16\% y el $50 \%{ }^{17,18}$. En nuestra serie fue del $17,5 \%$ y no hubo ningún caso de complicación, con una media de seguimiento de 4 años. Aunque la PIVB no se asocia con una mayor incidencia de complicaciones, no está exenta de consecuencias adversas. En primer lugar, se asocia con una mayor utilización de antibióticos $\mathrm{y}$, como se ha visto en nuestro estudio, su administración no parece ser necesaria en estos pacientes. Hasta donde nosotros sabemos, sólo hay un artículo publicado previamente en el que también se analiza el uso de antibióticos en pacientes con PIVB y la conclusión fue la misma ${ }^{6}$. La segunda consecuencia adversa que hemos visto es la mayor utilización de drenajes subhepáticos (34,6\% vs. 21\%). No hay ningún otro estudio en el que se analice este hecho, pero parece claro que es una práctica innecesaria. La tercera consecuencia adversa observada que hemos advertido es el aumento de la duración de la operación (una media de 12 minutos). Este hallazgo también fue observado por otros autores ${ }^{6,10,11}$, pero mientras en nuestro estudio sólo se puede atribuir al tiempo dedicado a lavar y aspirar la cavidad peritoneal, otros autores también lo relacionan con la menor experiencia del cirujano ${ }^{6}$. La última consecuencia adversa que hemos encontrado es el aumento significativo de la estancia posoperatoria, aunque las diferencias son pequeñas (2,6 y 2,4 días, respectivamente). Este hallazgo también fue publicado previamente por otros autores ${ }^{11}$.
Se han identificado varios factores que aumentan el riesgo de PIVB durante una CL electiva, tales como sexo varón ${ }^{10}$, edad avanzada ${ }^{6}$, experiencia del cirujano ${ }^{11}$, la historia de colecistitis aguda ${ }^{11}$, las laparotomías previas ${ }^{18}$ y la presencia de una pared vesicular engrosada ${ }^{18}$. Sin embargo, en un análisis multivariante llevado a cabo por Mohiuddin et al. ${ }^{19}$, sólo el sexo masculino, el antecedente de colecistitis aguda y la inflamación importante de la vesícula biliar eran factores de riesgo independientes. En nuestro estudio la incidencia de PIVB estaba significativamente aumentada en varones, pacientes con diagnóstico histológico de colecistitis aguda y pacientes con adherencias perivesiculares, pero no era mayor cuando el cirujano era residente.

La principal debilidad de este estudio es que se trata de una revisión retrospectiva, lo cual conlleva variabilidad en la actuación de los cirujanos, sobre todo en cuanto al uso de antibióticos, y la posibilidad de que no se hayan registrado casos de perforación iatrogénica de la vesícula biliar, lo cual genera sesgos en la interpretación de los resultados. Sin embargo, creemos que tiene algunas fortalezas, como la inclusión de un elevado número de pacientes y el análisis de un grupo de pacientes que no recibieron ningún antibiótico.

\section{Conclusión}

La PIVB, con vertido de bilis y/o cálculos hacia la cavidad peritoneal, no aumenta el riesgo de ISQ, pero se asocia con un aumento del uso de antibióticos y drenajes, así como una mayor duración de la cirugía y de la estancia posoperatoria.

\section{Responsabilidades éticas}

Protección de personas y animales. Los autores declaran que para esta investigación no se han realizado experimentos en seres humanos ni en animales.

Confidencialidad de los datos. Los autores declaran que en este artículo no aparecen datos de pacientes.

\section{Conflictos de interés: no hay}

Financiación: Este trabajo no recibió financiación ni becas, públicas o privadas. 


\section{Bibliografía}

1. Schäfer M, Suter C, Klaiber C, Werhrli H, Frei E, Krähenbühl L. Spilled gallstones after laparoscopic cholecystectomy. A relevant problem? A restrospective analysis of 10,174 laparoscopic cholecystectomies. Surg Endosc. 1998;12:305-9. DOI: 10.1007/ s004649900659.

2. Brockmann JG, Kocher T, Senninger NJ, Schurmann GM. Complications due to gallstones lost during laparoscopic cholecystectomy. Surg Endosc. 2002;16:1226-32. DOI:10.1007/s00464001-9173-8.

3. Demirbas BT, Gulluoglu BM, Aktan AO. Retained abdominal gallstones after laparoscopic cholecystectomy: a systematic review. Surg Laparosc Endosc Percutan Tech. 2015;25:97-9. DOI:10.1097/ SLE.0000000000000105

4. Woodfield JC, Rodgers M, Windsor JA. Peritoneal gallstones following laparoscopic cholecystectomy: incidence, complications and management. Surg Endosc. 2004;18:1200-7. DOI: 10.1007/ s00464-003-8260-4.

5. Nooghabi AJ, Hassanpour M, Jangjoo A. Consequences of lost gallstones during laparoscopic cholecystectomy: a review article. Surg Laparosc Endosc Percutan Tech. 2016;26:183-92. DOI:10.1097/ SLE.0000000000000274.

6. Barrat C, Champault A, Matthyssens L, Champault G. Iatrogenic perforation of the gallbladder during laproscopic cholecystectomy does not influence the prognosis. Prospective study. Ann
Chirg. 2004;129:25-9. DOI: 10.1016/j. anchir.2003.11.011.

7. Yildiz B, Abbasoglu O, Tirnaksiz B, Hamaloglu E, Ozdemir A, Sayek I. Determinants of postoperative infection after laparoscopic cholecystectomy. Hepatogastroenterology 2009;56:589-92.

8. Chang WT, Lee KT, Chuang SC, Wang SN, Kuo KK, Chen JS, et al. The impact of prophylactic antibiotics on postoperative infection complication in elective laparoscopic cholecystectomy: a prospective randomized study. Am J Surg. 2006;191:721-5. DOI: 10.1016/j. amjsurg.2006.01.050.

9. Kimura T, Goto H, Takeuchi Y, Yoshida M, Kobayashi T, Sakuramachi S, et al. Intra-abdominal contamination after gallbladder perforation during laparoscopic cholecystectomy and its complications. Surg Endosc. 1996;10:88891. DOI: $10.1007 / \mathrm{bf} 00188476$.

10. Usuba T, Nyumura Y, Takano Y, Lino T, Hanyu N. Clinical outcomes of laparoscopic cholecystectomy with accidental gallbladder perforation. Asian J Endosc Surg. 2017;10:162-5. DOI: 10.1111/ases.12348.

11. Aytaç B, Çakar S. The outcome of gallbladder perforation during laparoscopic cholecystectomy. Acta Chir Belg. 2003;103:388-91. DOI: $10.1080 / 00015458.2003 .11679449$

12. Horan TC, Andrus M, Dudeck MA. $\mathrm{CDC} / \mathrm{NHSN}$ surveillance definition of health care-associated infection and criteria for specific types of infections in the acute care setting. Am J Infect Control. 2008;36:309-32. doi: 10.1016/j. ajic.2008.03.002.
13. Soper NJ, Dunnegan DL. Does intraoperative gallbladder perforation influence the early outcome of laparoscopic cholecystectomy? Surg Laparosc Endosc. 1991;1:156-61.

14. Armiñanzas $C$, Herrera LA, Fariñas MC. Bacteriobilia: a nonresolved problem. Rev Esp Quimioter. 2016;29:113-8.

15. Choudhary A, Bechtold ML, Puli SR, Othman MO, Roy PK. Role of prophylactic antibiotics in laparoscopic cholecystectomy: A meta-analysis. J Gastrointest Surg. 2008;12:1847-53. doi: 10.1007/s11605-008-0681-x.

16. Sharma N, Garg PK, Hadke NS, Choudhary D. Role of prophylactic antibiotics in laparoscopic cholecystectomy and risk factors for surgical site infection: A randomized controlled trial. Surg Infect. 2010;11:36770. doi: 10.1089/sur.2008.084.

17. Zehetner J, Shamiyeh A, Wayand W. Lost gallstones in laparoscopic cholecystectomy: all possible complications. Am J Surg. 2007;193:73-8. DOI: 10.1016/j.amjsurg.2006.05.015.

18. De Simone P, Donadio R, Urbano D. The risk of gallbladder perforation at laparoscopic cholecystectomy. Surg Endosc. 1999;13:1099-102. DOI: 10.1007/ s004649901181.

19. Mohiuddin K, Nizami S, Fitzgibbons RJ, Watson P, Memon B, Memon MA. Predicting iatrogenic gallbladder perforation during laparoscopic cholecystectomy: a multivariate logistic regression analysis of risk factors. ANZ J Surg. 2006; 76:130-2. DOI: 10.1111/j.1445-2197.2006.03669.x 